\title{
Aplicación móvil para la gestión de pedidos online de servicios digitales de impresión, para incrementar la demanda de la empresa durante el confinamiento por covid-19
}

\author{
Mobile application for the management of online orders for digital printing \\ services, to increase the demand of the company during the confinement by covid-
} 19

\author{
Daniel O. Zúñiga-Vázquez ${ }^{a}$, Israel Acuña-Galván ${ }^{b}$,Evangelina Lezama-León ${ }^{c}$, Ernesto \\ Bolaños-Rodríguez ${ }^{d}$, Alonso E. Solis-Galindo ${ }^{e}$, Gaby Y. Vega Cano ${ }^{f}$
}

\begin{abstract}
:
The COVID-19 pandemic has not only brought problems, such as the disappearance of small businesses and the loss of employment, in many businesses it has been the opportunity they have long awaited to grow or implement improvements; Throughout the pandemic, a greater adaptability has been noted to face problems in what refers to a business, a clear example is the use of means such as mobile applications, web pages or social networks for the sale and purchase of products or otherwise advertise your business; The graphics industry, for example, has had a great impact with the measures taken to avoid contagion, since having companies promoting teleworking and schools implementing remote work, printing could decrease, however, this type of printing continues to be in demand today. service, therefore, a mobile application was made to cover the sales needs of a company that offers digital printing services, in order to serve the market, taking appropriate distancing measures and avoiding infections in these times.
\end{abstract}

Keywords:

covid-19, mobile application, printing online services

\section{Resumen:}

La pandemia del COVID-19 no solo ha traído problemas, como la desaparición de negocios pequeños y la pérdida de empleo, en muchos negocios ha sido la oportunidad que tanto esperaban para crecer o implementar mejoras; A lo largo de la pandemia se ha notado una mayor adaptabilidad para afrontar los problemas en lo que refiere a un negocio, un ejemplo claro es la utilización de medios como las aplicaciones móviles, las páginas web o redes sociales para la venta y compra de productos o de otro modo publicitar su negocio; La industria gráfica, por ejemplo, ha tenido un gran impacto con las medidas tomadas para evitar el contagio, ya que teniendo empresas fomentando el teletrabajo y escuelas implementando el trabajo a distancia la impresión podría disminuir, sin embargo, actualmente sigue teniendo demanda este tipo de servicio por ello se realizó una aplicación móvil que cubra las necesidades de venta de una empresa que ofrece servicios digitales de impresión, para así poder atender el mercado, tomando las medidas de distanciamiento oportunas y evitando contagios en estos tiempos.

\section{Palabras Clave:}

Covid-19, aplicación móvil, servicios de impresión en línea

\section{Introducción}

La pandemia del COVID-19 ha traído varios problemas a la sociedad, los cuales no estábamos preparados para recibir, por ende, muchas de las industrias han tenido que replantear su estructura y así poder sobrevivir, mientras que las empresas que no pudieron adaptarse posiblemente cerraron, otras en cambio encontraron un

\footnotetext{
a Universidad Autónoma del Estado de Hidalgo, https://orcid.org/0000-0001-5493-5846, Email: zu357662@uaeh.edu.mx

b Universidad Autónoma del Estado de Hidalgo, https://orcid.org/0000-0002-7714-0410, Email: israel_acuna4738@uaeh.edu.mx

e Universidad Autónoma del Estado de Hidalgo, https://orcid.org/0000-0003-0818-0897, Email: evangeli@uaeh.edu.mx

f Universidad Autónoma del Estado de Hidalgo, https://orcid.org/0000-0002-1432-7720, Email: ebolanos@uaeh.edu.mx

d Autor de Correspondencia, Universidad Autónoma del Estado de Hidalgo, https://orcid.org/0000-0002-3999-006X, Email: soliser@uaeh.edu.mx 
área de oportunidad para el desarrollo más amplio de su negocio, según [1] los negocios que se han adaptado a través de medios digitales han sobrevivido, incluso crecieron; Como es de esperar, los negocios se han enfrentado a nuevas y diferentes dificultades, en [3] clasificaron una lista de herramientas que ayudaría a los negocios de impresión, considerando la transición del servicio de forma presencial a ser online, si bien en [1] es posible percibir un cambio inesperado en negocios en general, hablando de la industria gráfica, en [2] se tenía una estimación de un crecimiento aproximado para la industria gráfica en los futuros años, debido a la pandemia estos datos han cambiado considerablemente puesto que esta no era un factor a tener en cuenta. En [2] podemos apreciar que en la industria gráfica las ventas han bajado hasta un $50 \%$, por ende, es necesario tomar medidas y actualizar los medios para poder llegar a un público aún más grande teniendo referencias de empresas como son Uber, DiDi y Beat en términos de transporte o UbearEats, Rappi, DiDi Food y Deliveroo en entrega de productos a domicilio, el transformar el negocio de la imprenta a los medios digitales podría ser un completo éxito, pues estas empresas e incluso el mercado online como son Amazon, AliExpress, Ebay y Mercado libre han crecido exponencialmente hasta un $150 \%$ durante el confinamiento según [4].

Si bien, la industria de impresión ha tenido un gran impacto durante el confinamiento, se espera que los avances post-pandemia sean aún mayores puesto que en [5] se relaciona esta industria con creaciones aún más avanzadas, implementando en sí lo que conocemos sobre robótica o electrónica, creando productos a través de la impresión misma y así maximizar sus áreas de impacto, como podría ser la creación de aparatos electrodomésticos o incluso artículos para la oficina implementando loT (Internet de las cosas; Internet of things) creando una variedad enorme sobre la cual se podría expandir esta industria. Sin embargo, esto es aún un vistazo al futuro ya que actualmente se deben presentar mejoras para optimizar la manera en cómo consumimos productos de imprenta, por esto mismo se realizó una aplicación móvil la cual facilitará la compra de productos de impresión.

Como sabemos debido a la pandemia se deben evitar lugares donde haya una gran concentración de gente esto hace que negocios de servicios de impresión no sean viables en ventas y disminuya su crecimiento, sin embargo, la realización de esta aplicación permitirá tanto a usuarios como a administradores trabajar de manera conjunta sin arriesgarse, pues con esta aplicación el usuario puede realizar un pedido que sea desde posters, impresiones, carteles o documentos enviando las especificaciones de su pedido y el archivo en cuestión, por otro lado el administrador encargado revisará el pedido a través de la misma aplicación, dando seguimiento y preparándolo. Una vez concluido, el administrador deberá validar la finalización de este mismo, y notificar al usuario que su pedido está listo, de esta manera solo necesitaría recurrir al local de servicios digitales de impresión para pagarlo y recogerlo.

Está claro que muchas empresas ofrecen algunos benéficos ya sea de compras por mayoreo o clientes frecuentes. En el negocio de impresión es más factible realizar pedidos en grupos que individuales, puesto que es más económico, por ello se generó una forma en que los usuarios pueden cargar saldo con el administrador o realizarlo de manera online para que esto abra paso a un descuento por mayoreo hacia los clientes, lo que incentiva a los usuarios a recargar saldo en la aplicación para obtener sus beneficios. En la figura 1 se muestra el concepto básico de como funcionaria la aplicación y que necesidades cubriría.

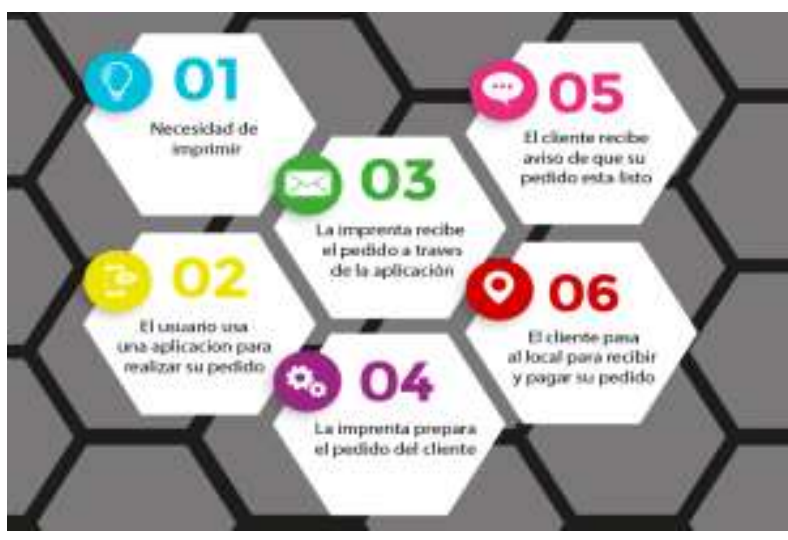

Figura 1. Funcionamiento básico de la aplicación

\section{Metodología}

A lo largo del proyecto se utilizó una metodología ágil usando un modelo Scrum, pues su facilidad por la adopción de cambios nuevos y la colaboración e interacción con el cliente, es una forma de asegurar buenos resultados en proyectos de este tipo. El contacto con el usuario también forma parte del proyecto, dando una retroalimentación de cada uno de los avances, lo que le permite al usuario experimentar el progreso del proyecto, además de sugerir las implementaciones 0 nuevas funcionalidades. Con el modelo scrum todos los implicados tiene conocimiento de que ocurre en el proyecto y cómo, además de tener inspecciones frecuentes para detectar lo posibles problemas, de esta manera es posible saber que el trabajo fluye y que el equipo funciona de forma auto-organizada. Cuando hay algo que cambiar, el equipo de trabajo se ajusta para 
alcanzar dicho objetivo, así este modelo se vuelve eficiente con proyectos de este tipo.

\section{Diseño de aplicación}

La aplicación será fácil de utilizar, pues su diseño hace que sea intuitivo tanto para el usuario como para el administrador, además de incluir una integración de envíos de correos electrónicos con la información necesaria. La aplicación facilita la manera en cómo solemos adquirir los servicios de impresión, en este caso, de pasar a estar la mayor parte del proceso dentro del local esperando a que el producto esté listo, ahora es posible hacerlo de forma digital, pues esta aplicación permite dar las especificaciones del producto, enviar el archivo a la empresa y recibir una notificación vía correo electrónico cuando nuestro pedido está listo, de esta manera ahorraríamos tiempo evitando estar en el establecimiento, y justo ahora, en tiempos donde la salud se ha visto afectada por la pandemia por COVID-19, estar en un establecimiento se ha vuelto peligroso, pues con esta aplicación también surge una solución en la cual las personas pueden seguir adquiriendo los productos de la industria de servicios digitales de impresión sin tener que arriesgar su salud. En la Figura 2 se muestra un diagrama de clases donde se puede observar cómo está conectada la aplicación, mostrando cómo están interconectadas las pantallas, sus características y datos que se manejan.

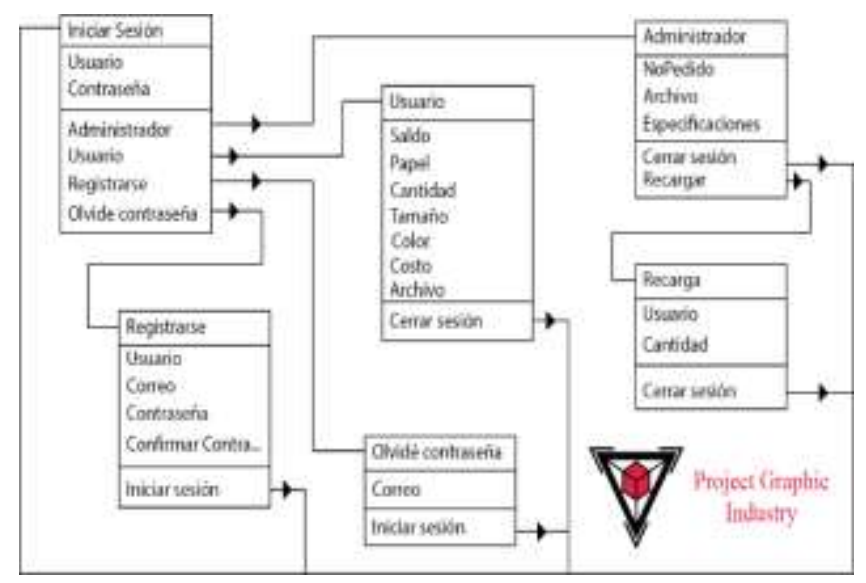

Figura 2. Diagrama UML del funcionamiento de la aplicación

En el diagrama podemos notar que la dirección de la información es lineal, pues para volver a alguna de las pantallas anteriores es necesario cumplir un ciclo, o bien repetir un paso para poder hacerlo. Es decir, si el usuario se encuentra en la pantalla usuario y cierra sesión, no será posible regresar a la pantalla de usuario a menos que inicie sesión nuevamente. En la Figura 3 se muestra un diagrama de casos de uso, en donde podemos observar cómo interactúa el usuario con la aplicación, una manera más simplificada del funcionamiento, aquí podemos ver como existen dos tipos de usuario, un administrador y un usuario común, el administrador se encargara de validar los pedidos, revisando las especificaciones, para después tenerlo listo y entregarlo al cliente, mientras que el usuario común solamente podrá interactuar con la pantalla que registra los pedidos, dando a su vez las especificaciones y el archivo para realizar su pedido con éxito, una vez su pedido esté listo, se enviará un correo electrónico (de forma automática) al usuario para notificarle que su pedido está listo y puede pasar a la sucursal para recogerlo.

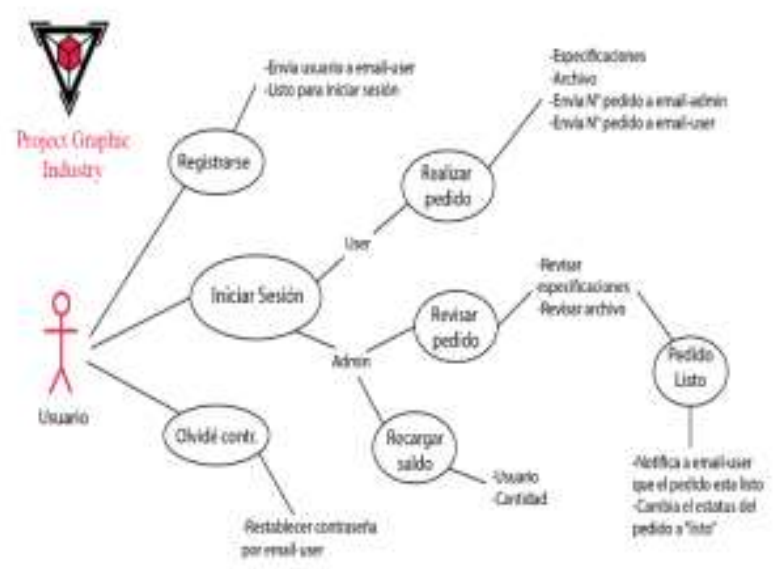

Figura 3. Diagrama de casos de uso

En la figura 4 se muestra un diagrama de secuencia del usuario, en el podemos observar que para poder realizar un pedido es necesario iniciar sesión.

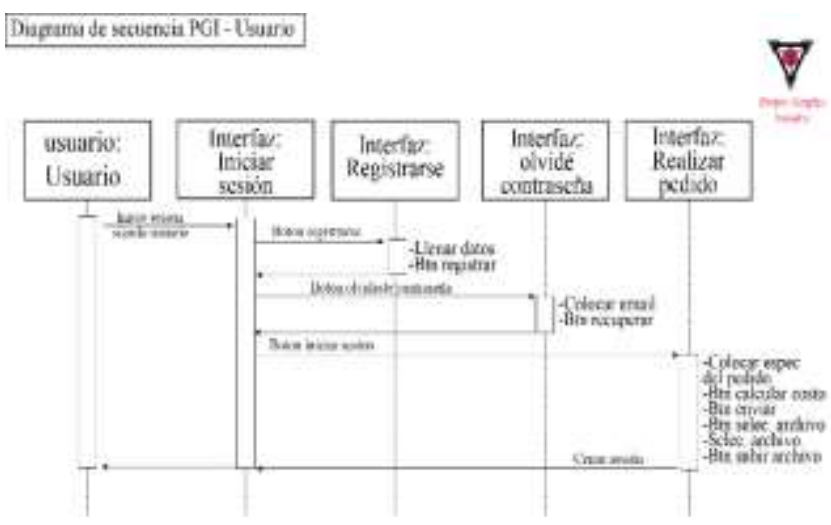

Figura 4. Diagrama de secuencia PGI usuario

Sí aún no se ha registrado es posible hacerlo mediante la pantalla para registrarse o bien, si el usuario olvidó sus contraseñas es posible recuperarlas a través de un correo en su respectiva pantalla, una vez hecho esto el usuario podrá iniciar sesión y comenzar a colocar las especificaciones del pedido, de igual manera cargar el archivo. Por otro lado, en la figura 5, se muestra el diagrama de secuencia del administrador, pues al igual 
que el usuario debe iniciar sesión, sin embargo, este solo tendrá la opción para revisar el pedido, descargar el respectivo archivo o bien realizar una recarga a un usuario en el establecimiento.

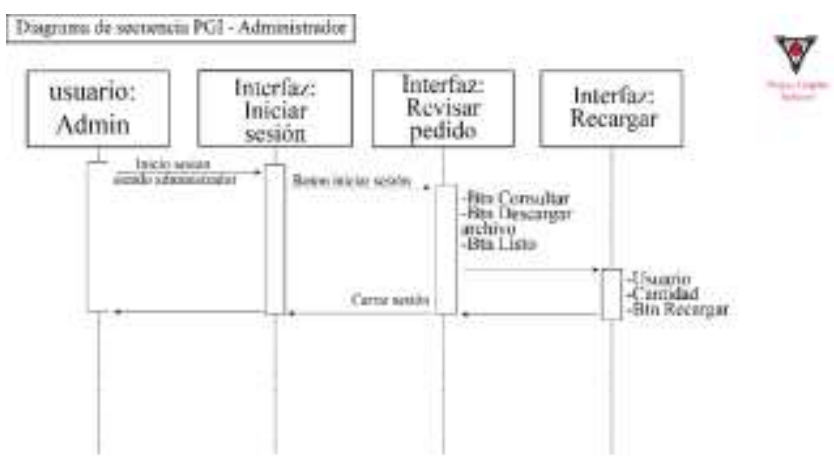

Figura 5. Diagrama de secuencia PGI - Administrador

\section{Resultados}

La aplicación facilita la gestión del servicio de impresión en línea lo que es un beneficio para la empresa y para el usuario de este servicio, pues gracias a su facilidad en la gestión de pedidos, es posible reducir tiempos de proceso. Al hacer una preventa de servicios el negocio se capitaliza y permite la fidelización del cliente. Con el manejo de esta App el cliente obtiene precios de mayoreo al pagar por adelantado. Esto también prevé un mayor número de clientes dándole un servicio eficiente e innovador. Desde el principio el usuario puede familiarizarse con el logo de la aplicación y a su vez iniciar en la primera pantalla, como se muestra en la Figura 6.

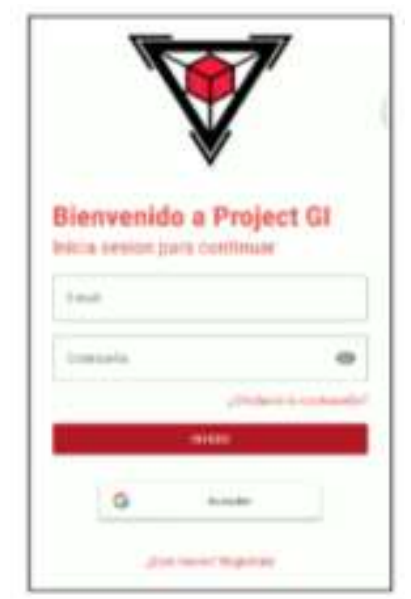

Figura 6. Pantalla inicio sesión

En esta pantalla el usuario deberá iniciar sesión, o, por el contrario, tendrá la facilidad de recuperar su contraseña con un botón que lo dirige a otra pantalla para recuperarla o bien registrarse, que de igual manera lo direccionará a un pequeño formulario para registrarse, el cual se muestra en la Figura 7. Una vez que nos hemos registrado se enviará un correo electrónico con nuestro nombre de Usuario, para poder revisar o recargar saldo. En la Figura 8, se observa la interfaz principal del usuario, en donde se colocan las especificaciones como son tipo de papel en el cual se hará el trabajo, la cantidad que se está solicitando, el tamaño de la impresión y si este será en color o en blanco y negro. Una vez se especifican todas estas características el usuario envía el pedido el cual genera un $\mathrm{N}^{\circ}$ de pedido, calcula el costo y lo resta del saldo actual del usuario, en caso de no tener saldo simplemente calcula el costo total, posterior a esto nos direcciona a una pantalla en la cual debemos seleccionar el archivo que vamos a cargar y este deberá subirse a la nube, en donde el administrador podrá revisarlo y descargarlo para posteriormente realizar el pedido. Una vez hecho esto, la aplicación envía dos correos, un correo al usuario con el $\mathrm{N}^{\circ}$ de pedido que se generó y otro correo al administrador, notificando que se realizó un pedido, mostrando que $\mathrm{N}^{\circ}$ de pedido tiene, al finalizar la carga del archivo, se direcciona al usuario a la pantalla principal del usuario, por si quiere realizar otro pedido o bien cerrar sesión.

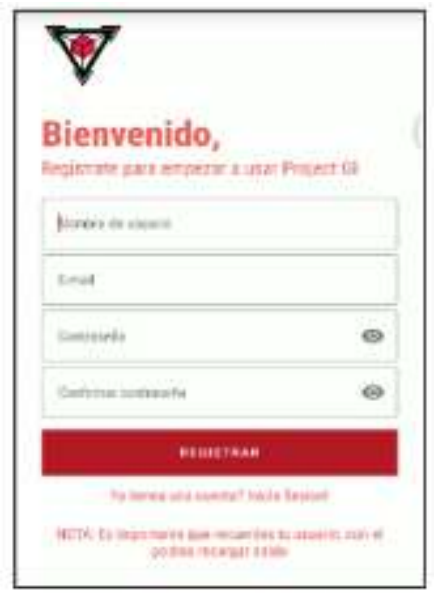

Figura 7. Pantalla registro

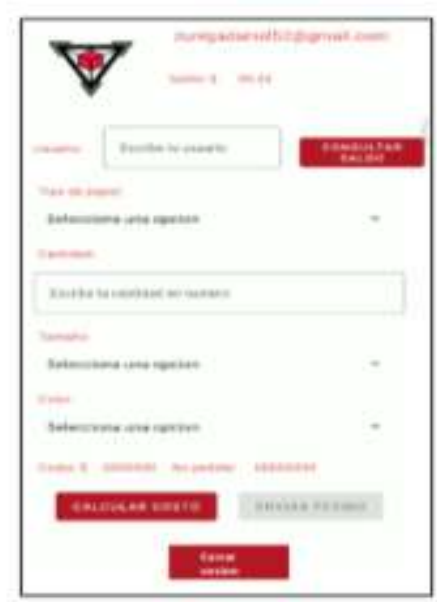

Figura 8. Pantalla Usuario 


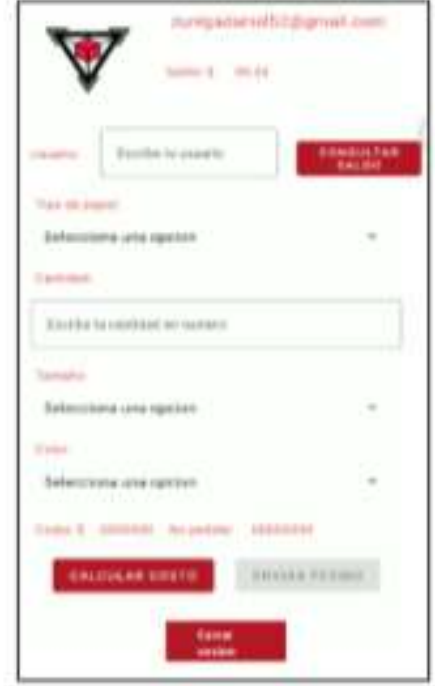

Figura 9. Pantalla Administrador

Por otro lado, el administrador no necesita registrarse, pues este ya está definido en el sistema. Sin embargo, el administrador igual deberá iniciar sesión, pero se le direcciona a la pantalla de administrador como se muestra en la Figura 9, ahí podrá consultar algún pedido con su respectivo $\mathrm{N}^{\circ}$ de pedido que se envió al correo electrónico de administrador, también podrá descargar el archivo correspondiente al pedido y eliminar el pedido de la lista de pendientes una vez este haya finalizado, también tiene un botón para realizar recargas a los usuarios o bien, para cerrar sesión. En la Figura 10 es posible observar el formato de los correos electrónicos que llegar al usuario, con su respectiva información, ya sea al registrarse con su nombre de usuario, cuando ha realizado un pedido o bien cuando su pedido está listo y puede pasar a recogerlo.

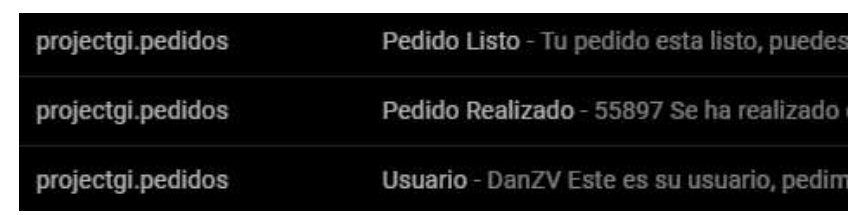

Figura 10. Correos

Al desarrollar la aplicación se debía tener en cuenta el crear una interfaz de usuario que facilite el manejo de la aplicación, así como la accesibilidad que tendría el público para poder adquirir estos servicios. Para el usuario es más sencillo manipular los datos y enviar un pedido $\sin$ necesidad de estar en el local. Para los administradores es más eficiente la gestión de pedidos pues, se tiene un orden de llegada de las órdenes de trabajo, además permite saber cuántos pedidos están pendientes y cuantos han sido completados. Si bien, estos servicios digitales se habían implementado en servicios de transporte o comida teniendo un impacto positivo en la sociedad, se prevé que la industria gráfica no será la excepción, pues con aplicaciones de esta naturaleza que apoyen al mercado gráfico, es posible que las ventas puedan volver a su crecimiento habitual en tiempos donde cualquier actividad fuera de casa es riesgosa.

\section{Conclusión}

Existen diferentes alternativas para la compra y venta del material impreso, sin embargo, no todas están tan extendidas 0 son usadas en ciertas localidades por ciertos negocios, por eso la realización de esta aplicación se usa para beneficio de un negocio, impulsando su crecimiento. Con pequeños negocios implementando mejoras y actualizando sus servicios de forma digital es posible que la industria gráfica tenga una gran oportunidad de crecimiento en diversas áreas y abarcando un gran mercado; a pesar de haber sufrido declives a inicios de la pandemia las implementaciones han abierto paso a oportunidades nuevas, que antes parecían muy lejanas.

\section{Referencias}

[1] Sulbarán Lovera, P. (08 de mayo de 2020). Economía y coronavirus: 7 emprendimientos de Latinoamérica que se reinventaron en medio de la pandemia y están prosperando. Obtenido de BBC News Mundo: https://www.bbc.com/mundo/noticias-america-latina-52495847

[2] Sanz, J. R. (17 de febrero de 2020). It User Centro de Recursos. Obtenido de La industria mundial de la impresión está en una etapa de transición: https://impresiondigital.ituser.es/noticias-yactualidad/2020/02/la-industria-mundial-de-la-impresion-esta-en-unaetapa-de-transicion

[3] Malone-McGrew, J. (23 de marzo de 2021). 5 formas en que las herramientas de impresión de post composición le conducirán al éxito en 2021. Obtenido de Canales sectoriales Interpresas: https://www.interempresas.net/Graficas/Articulos/346249-5-formasque-herramientas-impresion-poscomposicion-le-conduciran-al-exito2021.html

[4] Núñez, J. (17 de septiembre de 2020). EL PAIS. Obtenido de ¿Qué tipos de negocios sobrevivirán al coronavirus?: https://elpais.com/economia/2020/08/20/nuevos_tiempos/1597919911 _259535.html

[5] RedacciónMCP. (08 de agosto de 2018). MuyComputerPro. Obtenido de Cómo será el negocio de la impresión en 2025: https://www.muycomputerpro.com/2018/08/08/negocio-impresion2025 